\title{
Avaliação da cobertura da triagem auditiva neonatal nas macrorregiões de saúde do estado da Bahia entre os anos de 2011 a 2018
}

\author{
Evaluation of the coverage of the newborn hearing screening in the health macro- \\ regions of the state of Bahia between the years 2011 to 2018 \\ Morenah Gomes Lôbo ${ }^{1}$, Caio Leônidas Oliveira de Andrade², Crésio Alves ${ }^{3 *}$ \\ ${ }_{1}^{1}$ Programa de Pós-graduação em Processos Interativos dos Órgãos e Sistemas, Instituto de Ciências da Saúde, \\ Universidade Federal da Bahia (UFBA); ' Docente de Fonoaudiologia do Departamento de Ciências da Vida \\ da Universidade do Estado da Bahia (UNEB) ${ }^{3}$ Departamento de Pediatria, Faculdade de Medicina da Bahia, \\ Universidade Federal da Bahia (UFBA) - Salvador (BA), Brasil.
}

\begin{abstract}
Resumo
Introdução: a triagem auditiva neonatal (TAN) está disposta na Lei no 12.303/2010, a qual, obriga a realização do teste da orelhinha em todos os hospitais e maternidades do Brasil. Porém, vários estados brasileiros ainda não atingiram o nível de cobertura satisfatória desse programa conforme preconizado. Objetivo: analisar a cobertura da triagem auditiva neonatal no período entre 2011 a 2018 nas macrorregiões do Estado da Bahia. Metodologia: estudo ecológico, quase-experimental, realizado por meio do tabulador genérico de domínio público desenvolvido pelo DATASUS. Resultados: a produção ambulatorial dos procedimentos da TAN no Estado da Bahia obteve crescimento considerável ao longo do período do estudo, porém os achados apontaram que as taxas de crescimentos anuais apresentaram um comportamento irregular no seu crescimento. Observou-se que a média das taxas de cobertura na Bahia é inferior à média nacional e muito discrepante quando comparada a média universal. Conclusão: os achados demonstraram que o Estado da Bahia segue com taxas de cobertura da TAN inferior ao preconizado pelo COMUSA (Comitê Multiprofissional de Saúde Auditiva), apresentando distribuição irregular na realização dos procedimentos, ausência de recursos tecnológicos em algumas macrorregiões, o que compromete o princípio da universalidade da TAN no Estado .

Palavras-chaves: Cobertura de saúde. Emissões otoacústicas. Potenciais evocados auditivos. Qualidade. Acesso e avaliação da assistência à saúde. Recém-nascido.
\end{abstract}

\begin{abstract}
Introduction: the newborn hearing screening (NHS) is set out in Law № 12.303/2010, which requires the ear test to be performed in all hospitals and maternity hospitals in Brazil. However, several Brazilian states have not yet reached the level of satisfactory coverage of this program as recommended. Objective: to analyze the coverage of newborn hearing screening, between 2011 and 2018, in the macro-regions of the State of Bahia. Methodology: ecological, quasi-experimental study, carried out using the generic public domain tab developed by DATASUS. Results: the NHS procedures in the State of Bahia achieved considerable growth over the period of the study, however the findings pointed out that the annual growth rates showed an unstable behavior in their growth. It was observed that the average coverage rates in Bahia are lower than the national average and very different when compared to the universal average. Conclusion: the findings showed that the State of Bahia continues to have NHS coverage rates lower than recommended by COMUSA (Multiprofessional Hearing Health Committee), unstable distribution in the performance of procedures, there are no technological resources in some macro-regions, which compromises the principle of universality of NHS in the state. Keywords: Health coverage. Otoacoustic emissions. Auditory evoked potentials. Health care quality. Access, and evaluation. Newborn.
\end{abstract}

\section{INTRODUÇÃO}

A Triagem Auditiva Neonatal (TAN) foi uma conquista para os direitos e cuidados da saúde do recém-nascido adquirido através da Lei no $12.303 / 2010$ que dispõe sobre a obrigatoriedade da realização do teste orelhinha (emissões otoacústicas), em todos os hospitais e maternidades do Brasil, nas crianças nascidas em suas dependências (BRASIL, 2010).

Correspondente/Corresponding: *Crésio Alves - Instituto de Ciências da Saúde/UFBA. - End: Av. Reitor Miguel Calmon, s/n, Vale do Canela. 40110-100. Salvador, Ba. - Tel: (71) 99178-4055 - E-mail: cresio.alves@ uol.com.br
De acordo com as Diretrizes de Atenção da Triagem Auditiva Neonatal, a TAN baseia-se no teste e retestes que estão fundamentadas nos parâmetros fisiológicos e eletroacústicos da audição, tendo como finalidade a identificação prévia de possíveis alterações auditivas em neonatos e lactantes (BRASIL, 2012).

Nesse sentido, a TAN prioriza o diagnóstico das perdas auditivas até o terceiro mês de vida e, quando necessário, o início da intervenção do tratamento até o sexto mês a fim de contemplar o período de máxima plasticidade do sistema nervoso central (BRASIL, 2012; CFF, 2016) para minimizar ou dirimir os prejuízos no desenvolvimento das habilidades de comunicação (SBP, 2018). 
Embora a TAN tenha demonstrado sua importância nos programas e políticas públicas de saúde, bem como se tenha verificado um aumento do número de seus procedimentos no Brasil nos últimos anos, a cobertura ainda é ineficiente, não contemplando o princípio básico da universalidade do Sistema Único de Saúde (SUS), especialmente em regiões socialmente menos favorecidas como o Nordeste (CRUZ; FERRITE, 2014).

Adicionalmente, verifica-se que os Estados do Nordeste com maior expansão territorial, a exemplo da Bahia, têm apresentado índices de TAN abaixo do preconizado, o que pode estar associado com a falta de fonoaudiólogos no SUS, insuficiência de centros de referências em saúde auditiva e limitada quantidade de maternidades públicas com o programa da TAN (DIAS; PASCHOAL; CAVALCANTI, 2017).

Em 2019, o Conselho Regional de Fonoaudiologia da 4a região, alertou através de um debate com o poder público sobre a importância da TAN no município de Salvador bem como no Estado da Bahia. Essa manifestação baseou-se no fato das fiscalizações do órgão profissional encontrar durante as suas visitas técnicas, em várias maternidades, a não realização da TAN por causas diversas como, por exemplo, equipamentos danificados e falta de fonoaudiólogos.

Face ao exposto, o presente artigo tem como objetivo realizar uma análise da cobertura da triagem auditiva neonatal nas diferentes macrorregiões do Estado da Bahia após a vigência da lei no 12.303/2010.

\section{METODOLOGIA}

Trata-se de um estudo ecológico, quase-experimental realizado por meio do tabulador genérico de domínio público desenvolvido pelo DATASUS (Departamento de Informática do Sistema Único de Saúde do Brasil), o TABNET, dados estes vinculado ao Ministério da Saúde, no endereço eletrônico: http://www.datasus.saude.gov. br, para gerar informações das bases de dados.

Para a construção desta pesquisa foi selecionado no tabulador como critério de inclusão o grupo de produção ambulatorial (SIA/SUS), por local de atendimento a partir de 2008, escolhendo como abrangência geográfica o Estado da Bahia. Para a formação da tabela que consta nas análises deste estudo inclui como variáveis quantidade aprovada por ano atendimento segundo macrorregião dos procedimentos audiológicos emissões otoacústicas para triagem auditiva (0211070149) e potencial evocado auditivo para triagem auditiva (0211070270).

Buscou-se no Sistema de Informações de Beneficiários (SIB) da Agência Nacional de Saúde Suplementar (ANS) a frequência absoluta das assistências suplementares de saúde, por município do Estado da Bahia, entre o período de 2011 a 2018, por meio do quantitativo de crianças beneficiária com até 1 ano de idade a fim de excluí-las da população usuária do SUS, no intuito de tornar os dados o mais próximo da realidade local e contemplar os objetivos do estudo.
Fluxograma 1- Etapas para a realização da coleta de dados no sistema de informação ambulatorial do SUS.

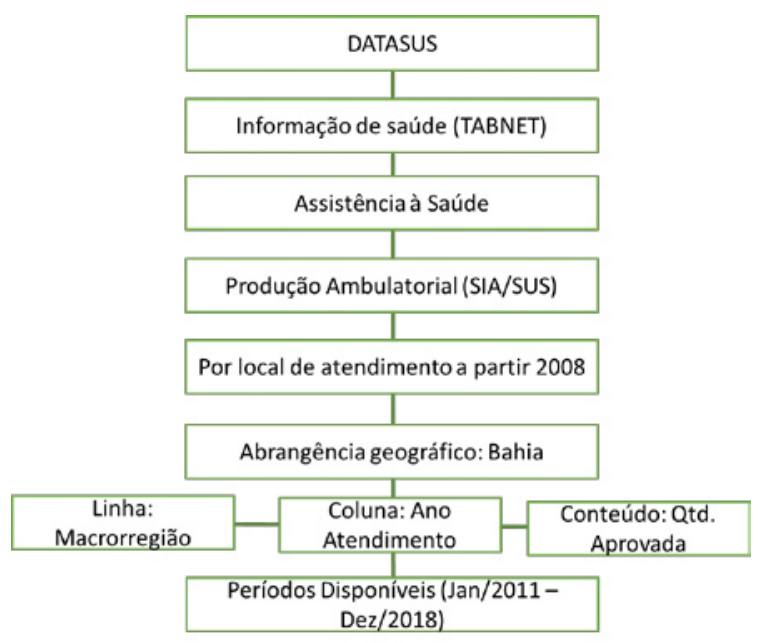

Fonte: Autoria própria

As taxas de cobertura da TAN foram calculadas por intermédio da seguinte fórmula matemática: Taxa de cobertura da TAN = nTA / nNV - nPS x 100 (nTA equivale a produção ambulatorial das emissões otoacústicas transientes (EOAT) somado ao quantitativo do potencial evocado auditivo de tronco encefálico automático (PEATE-A) aprovados para a TAN pelo SUS, nNV corresponde ao quantitativo de Nascidos Vivos disponibilizados no SINASC e o nPS equivale aos usuários da assistências suplementares de saúde com até um ano de idade).

Os critérios dos não incluídos foram adotados para todos os procedimentos audiológicos que não compõem a bateria de exames destinados a TAN, bem como a produção ambulatorial de outros Estados da federação.

O estudo por apresentar um caráter de análise de dados secundários, disponíveis em plataforma de domínio público, não foi submetido e registrado no sistema do CEP/ CONEP conforme a Resolução do Conselho Nacional de Saúde no510/2016, porém todos os princípios éticos envolvidos na análise dos dados foram cuidadosamente respeitados.

Os dados foram tabulados no TABWIN, um programa criado e disponibilizado pelo DATASUS, e analisados de forma descritiva por meio de medidas de tendência central, medidas de dispersão, tamanho do efeito, valores absolutos e relativos, intervalos interquartis e percentis.

\section{RESULTADOS}

Os dados obtidos através do SIA/SUS e expostos na tabela 1 demostraram que após a aprovação da lei no12.303/2010, a TAN no Estado da Bahia obteve crescimento considerável ao longo dos oito anos que sucedem. No entanto, os achados apontam que as taxas de crescimentos anuais apresentaram um comportamento irregular no seu crescimento. Ainda conforme a tabela 1 , nota-se que os procedimentos de EOA foram responsáveis por mais de $90 \%$ da produção ambulatorial. 
Tabela 1 - Distribuição dos valores absolutos, relativos e taxa de crescimento anual dos exames de triagem auditiva neonatal realizados no Estado da Bahia no período de 2011-2018.

\begin{tabular}{rrrrrrr}
\hline Ano & EOA & $\%$ & PEATE-A & $\%$ & Total & $\begin{array}{c}\text { TX. } \\
\text { Cres. }\end{array}$ \\
\hline 2011 & 20606 & 91,6 & 1891 & 8,4 & 22497 & $0^{*}$ \\
2012 & 24462 & 99,3 & 166 & 0,7 & 24628 & 9,5 \\
2013 & 19451 & 99,5 & 92 & 0,5 & 19543 & $-20,6$ \\
2014 & 27957 & 98,4 & 460 & 1,6 & 28417 & 45,4 \\
2015 & 37860 & 97,8 & 833 & 2,2 & 38693 & $-36,2$ \\
2016 & 34113 & 96,8 & 1115 & 3,2 & 35228 & $-8,9$ \\
2017 & 33393 & 95,2 & 1701 & 4,8 & 35094 & $-0,4$ \\
2018 & 35216 & 97,2 & 1022 & 2,8 & 36238 & 3,3 \\
\hline
\end{tabular}

Legenda: EOA - Emissões otoacústicas / PEATE-A - Potenciais evocados auditivo do tronco encefálico automático/Tx. Cresc. Taxa de crescimento anual/ (*) Ano inicial da análise das taxas de crescimento.

Fonte: Sistema de Informação Ambulatorial (SIA/SUS).
Analisando o comportamento da TAN nas diferentes macrorregiões de saúde da Bahia, evidencia-se ausência de registros de procedimento do PEATE nas macrorregiões de saúde Sul (NRS - Ilhéus) e Norte (NRS - Juazeiro). Ainda de acordo com os dados, pode-se verificar que somente a macrorregião de saúde Leste (NRS - Salvador) apresentou registro de realização de ambos os procedimentos da TAN no decorrer de todo período estudado, sendo também a macrorregião de saúde com maior frequência de exames realizados quando comparado as demais macrorregiões estudadas. (tabela 2)

Observa-se ainda que o número de nascidos vivos é superior a quantidade de procedimentos da TAN realizados em todas as macrorregiões de saúde na Bahia, bem como ao longo do período 2011 a 2018.

\begin{tabular}{|c|c|c|c|c|c|c|c|c|c|c|c|c|c|c|c|c|c|}
\hline \multirow{3}{*}{$\begin{array}{l}\text { Macrorregião } \\
\text { de saúde da } \\
\text { Bahia }\end{array}$} & \multirow{3}{*}{ Registro } & \multicolumn{16}{|c|}{ Taxa de cobertura dos procedimentos da TAN } \\
\hline & & \multicolumn{2}{|c|}{2011} & \multicolumn{2}{|c|}{2012} & \multicolumn{2}{|c|}{2013} & \multicolumn{2}{|c|}{2014} & \multirow{2}{*}{$\begin{array}{c}2015 \\
\mathrm{n}\end{array}$} & \multirow[b]{2}{*}{$\mathrm{Tx}(\%)$} & \multicolumn{2}{|c|}{2016} & \multicolumn{2}{|c|}{2017} & \multicolumn{2}{|c|}{2018} \\
\hline & & $\mathrm{n}$ & $\mathrm{Tx}(\%)$ & $\mathrm{n}$ & $T x(\%)$ & $\mathrm{N}$ & $\mathrm{Tx}(\%)$ & $\mathrm{n}$ & $\mathrm{Tx}(\%)$ & & & $\mathrm{N}$ & $\mathrm{Tx}(\%)$ & $\mathrm{n}$ & $\mathrm{Tx}(\%)$ & $n$ & $\mathrm{Tx}(\%)$ \\
\hline Sul & PEATE & 0 & & 0 & & 0 & & 0 & & 0 & & 0 & & 0 & & 0 & \\
\hline \multirow[t]{2}{*}{ (Ilhéus) } & EOA & 16 & 0,06 & 129 & 0,53 & 129 & 0,55 & 981 & 4,34 & 1.775 & 7,61 & 1.636 & 7,34 & 4.551 & 19,83 & 5.897 & 25,85 \\
\hline & SINASC & 25.164 & & 24.301 & & 23.318 & & 22.569 & & 23.309 & & 22.269 & & 22.946 & & 22.811 & \\
\hline Sudoeste & PEATE & 0 & & 0 & & 0 & & 0 & & 0 & & 8 & & 0 & & 0 & \\
\hline \multirow[t]{2}{*}{$\begin{array}{l}\text { (Vitória da } \\
\text { Conquista) }\end{array}$} & EOA & 0 & 0,0 & 0 & 0,0 & 112 & 0,48 & 680 & 2,92 & 2.532 & 10,8 & 3.064 & 13,5 & 2.379 & 10,28 & 2.910 & 12,55 \\
\hline & SINASC & 24.302 & & 23.811 & & 23.036 & & 23.270 & & 23.529 & & 22.741 & & 23.146 & & 23.178 & \\
\hline Oeste & PEATE & 0 & & 0 & & 0 & & 0 & & 0 & & 0 & & 0 & & 0 & \\
\hline \multirow[t]{2}{*}{ (Barreiras) } & EOA & 2.737 & 18,81 & 3.883 & 27,53 & 4.074 & 28,48 & 2.590 & 17,7 & 3.581 & 24,9 & 1.425 & 10,2 & 683 & 4,70 & 2.154 & 14,49 \\
\hline & SINASC & 14.678 & & 14.264 & & 14.439 & & 14.682 & & 14.504 & & 14.067 & & 14.673 & & 15.012 & \\
\hline Norte & PEATE & 0 & & 0 & & 0 & & 0 & & 0 & & 0 & & 0 & & 0 & \\
\hline \multirow[t]{2}{*}{ (Juazeiro) } & EOA & 3.766 & 21,60 & 4.063 & 23,93 & 3.163 & 0,0 & 4.613 & 27,6 & 4.385 & 25,30 & 4.284 & 26,1 & 4.958 & 28,86 & 5.399 & 30,40 \\
\hline & SINASC & 17.422 & & 16.976 & & 16.546 & & 16.705 & & 17.328 & & 16.406 & & 17.174 & & 17.762 & \\
\hline Nordeste & PEATE & 0 & & 0 & & 0 & & 0 & & 0 & & 0 & & 24 & & 67 & \\
\hline \multirow[t]{2}{*}{ (Alagoinhas) } & EOA & 109 & 0,91 & 1.364 & 11,85 & 2.121 & 19,36 & 2.670 & 24,6 & 3.099 & 29,4 & 2.827 & 26,7 & 2.062 & 19,49 & 2.745 & 25,94 \\
\hline & SINASC & 11.871 & & 11.505 & & 10.958 & & 10.861 & & 10.555 & & 10.577 & & 10.706 & & 10.848 & \\
\hline Leste & PEATE & 1.863 & & 147 & & 46 & & 460 & & 833 & & 1.107 & & 1.676 & & 948 & \\
\hline \multirow[t]{2}{*}{ (Salvador) } & EOA & 4.834 & 10,59 & 6.087 & 10,03 & 3.434 & 5,74 & 9.219 & 16 & 11.629 & 20,3 & 9.279 & 17,60 & 8.553 & 18,06 & 6.527 & 15,1 \\
\hline & SINASC & 63.422 & & 62.709 & & 61.464 & & 61.484 & & 61.937 & & 60.066 & & 60.194 & & 59.881 & \\
\hline Extremo Sul & PEATE & 28 & & 19 & & 46 & & 0 & & 0 & & 0 & & 1 & & 0 & \\
\hline \multirow[t]{2}{*}{$\begin{array}{l}\text { (Teixeira } \\
\text { Freitas) }\end{array}$} & EOA & 1.337 & 10,16 & 1.770 & 13,39 & 666 & 5,67 & 1.501 & 11,6 & 3.283 & 25 & 2.785 & 22,1 & 1.019 & 7,82 & 1.905 & 15,1 \\
\hline & SINASC & 13.432 & & 13.372 & & 12.554 & & 12.950 & & 13.134 & & 12.614 & & 13.031 & & 13.498 & \\
\hline Centro Leste & PEATE & 0 & & 0 & & 0 & & 0 & & 0 & & 0 & & 0 & & 0 & \\
\hline \multirow[t]{2}{*}{$\begin{array}{l}\text { (Feira } \\
\text { Santana) }\end{array}$} & EOA & 7.807 & 24,14 & 7.165 & 22,83 & 5.752 & 19,1 & 5.703 & 18,8 & 7.576 & 24,5 & 6.991 & 23,4 & 6.858 & 22,53 & 5.857 & 19,83 \\
\hline & SINASC & 32.341 & & 31.380 & & 30.117 & & 30.406 & & 30.939 & & 29.910 & & 30.537 & & 30.510 & \\
\hline Centro Norte & PEATE & 0 & & 0 & & 0 & & 0 & & 0 & & 0 & & 0 & & 7 & \\
\hline \multirow[t]{3}{*}{ (Jacobina) } & EOA & 0 & 0,0 & 1 & 0,008 & 0 & 0,0 & 0 & 0,0 & 0 & 0,0 & 1.822 & 16,3 & 2.330 & 19,95 & 1.822 & 15,46 \\
\hline & SINASC & 12.388 & & 11.672 & & 10.889 & & 11.078 & & 11.408 & & 11.166 & & 11.679 & & 11.829 & \\
\hline & PEATE & 1.891 & & 166 & & 92 & & 460 & & 833 & & 1.115 & & 1.701 & & 1.022 & \\
\hline \multirow[t]{2}{*}{ Bahia } & EOA & 20.606 & 10,48 & 24.462 & 11,77 & 19.451 & 9,66 & 27.657 & 13,9 & 37.860 & 18,8 & 34.113 & 17,6 & 33.393 & 17,52 & 35.216 & 18,70 \\
\hline & SINASC & 215.020 & & 209.990 & & 203.321 & & 204.005 & & 206.643 & & 199.816 & & 204.086 & & 205.329 & \\
\hline
\end{tabular}

Legenda: EOA - Emissões otoacústicas / PEATE - Potenciais evocados auditivo do tronco encefálico / NRS - Núcleo regionais de saúde /Tx. Taxa de cobertura em porcentagem da TAN/TAN - Triagem auditiva neonatal/SINASC - Sistema de Informação de Nascidos Vivos.

Fonte: Sistema de Informação Ambulatorial (SIA/SUS) e Sistema de Informação de Nascidos Vivos. 
O gráfico 1 ilustra a média anual da taxa de cobertura da TAN no Estado da Bahia e nas suas respectivas macrorregiões de saúde em comparação com as taxas nacional e universal. Observa-se que a média das taxas de cobertura na Bahia é inferior à média nacional e muito discrepante quando comparada à média universal. Dentre as macrorregiões de saúde, verificou que o Norte e Centro Leste obtiveram as maiores médias do Estado, sendo superior até à macrorregião onde fica sediada à capital do estado (Leste).

Gráfico 1 - Distribuição da média anual das taxas de cobertura da TAN nas macrorregiões da Bahia no período de 2011-2018 em comparação a taxa universal e nacional.

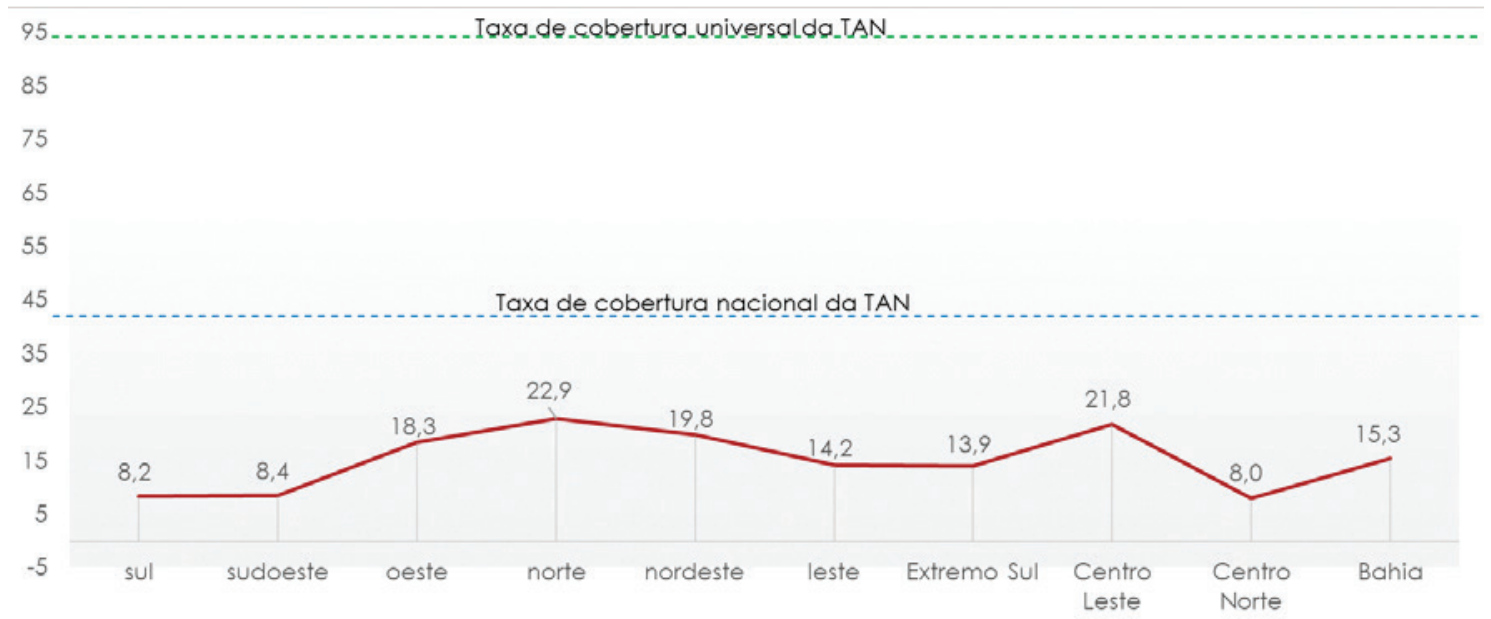

Fonte: Autoria Própria

Legenda: TAN - triagem auditiva neonatal

Na tabela 3 verifica-se que a quantidade de equipamento das emissões otoacústicas transientes (EOAT) é insuficiente para a demanda populacional. A macrorregião de saúde Leste (NRS - Salvador) se destaca por possuir a maior parcela do quantitativo dos equipamentos quando comparada as outras macrorregiões, sendo as macrorregiões do Sudoeste - (NBS - Vitória da Conquista), Norte
(NRS - Juazeiro) e Centro-Norte (NRS - Jacobina) as que obtiveram os menores quantitativos de equipamentos registrados.

Tabela 3 - Número de equipamentos das emissões otoacústicas transientes por ano nas macrorregiões do Estado da Bahia no período de 2011-2018.

\begin{tabular}{lcccccccc}
\hline \multicolumn{1}{c}{ Macrorregião de Saúde } & $\mathbf{2 0 1 1}$ & $\mathbf{2 0 1 2}$ & $\mathbf{2 0 1 3}$ & $\mathbf{2 0 1 4}$ & $\mathbf{2 0 1 5}$ & $\mathbf{2 0 1 6}$ & $\mathbf{2 0 1 7}$ & $\mathbf{2 0 1 8}$ \\
\hline Sul - (NBS - Ilhéus) & 2 & 2 & 2 & 3 & 6 & 6 & 6 & 6 \\
Sudoeste (NBS - Vitória da Conquista) & 0 & 1 & 1 & 1 & 1 & 1 & 1 & 1 \\
Oeste (NBS - Barreiras) & 0 & 1 & 1 & 1 & 1 & 1 & 2 & 2 \\
Norte (NRS - Juazeiro) & 1 & 1 & 1 & 1 & 1 & 1 & 1 & 1 \\
Nordeste (NRS - Alagoinhas) & 1 & 1 & 1 & 1 & 0 & 0 & 1 & 2 \\
Leste (NRS - Salvador) & 1 & 6 & 7 & 7 & 9 & 12 & 11 & 14 \\
Extremo Sul (NRS - Teixeira Freitas) & 1 & 1 & 1 & 1 & 1 & 2 & 3 & 3 \\
Centro Leste (NRS - Feira Santana) & 0 & 0 & 1 & 2 & 3 & 3 & 3 & 2 \\
Centro Norte (NRS - Jacobina) & 0 & 1 & 1 & 1 & 1 & 1 & 1 & 1 \\
\hline
\end{tabular}

NRS - Núcleo regionais de saúde /TAN - Triagem auditiva neonatal.

Fonte: Sistema de Informação Ambulatorial (SIA/SUS).

Já na tabela 4 consta o quantitativo dos equipamentos das emissões otoacústicas por produto de distorção (EOAPD) nas diferentes macrorregiões da Bahia. Nota-se que a macrorregião Norte (NRS - Juazeiro) é a única com ausência de registro deste tipo de equipamento e a macrorregião de saúde do Sudoeste (NBS - Vitória da Conquista) não possui equipamentos de EOAPD. Por outro lado, a macrorregião Leste (NRS-Salvador) tem o maior quantitativo desses equipamentos. 
Tabela 4 - Número de equipamentos das emissões otoacústicas por produto de distorção por ano nas macrorregiões do Estado da Bahia no período de 2011-2018.

\begin{tabular}{lcccccccc}
\hline \multicolumn{1}{c}{ Macrorregião de Saúde } & $\mathbf{2 0 1 1}$ & $\mathbf{2 0 1 2}$ & $\mathbf{2 0 1 3}$ & $\mathbf{2 0 1 4}$ & $\mathbf{2 0 1 5}$ & $\mathbf{2 0 1 6}$ & $\mathbf{2 0 1 7}$ & $\mathbf{2 0 1 8}$ \\
\hline Sul - (NBS - Ilhéus) & 2 & 3 & 3 & 2 & 4 & 4 & 4 & 4 \\
Sudoeste (NBS - Vitória da Conquista) & 0 & 0 & 0 & 0 & 0 & 0 & 0 & 0 \\
Oeste (NBS - Barreiras) & 0 & 1 & 2 & 2 & 2 & 2 & 3 & 3 \\
Norte (NRS - Juazeiro) & 0 & 0 & 0 & 0 & 0 & 0 & 0 & 0 \\
Nordeste (NRS - Alagoinhas) & 0 & 0 & 0 & 0 & 0 & 0 & 1 & 1 \\
Leste (NRS - Salvador) & 1 & 4 & 4 & 4 & 5 & 6 & 5 & 7 \\
Extremo Sul (NRS - Teixeira Freitas) & 1 & 1 & 1 & 1 & 1 & 2 & 3 & 2 \\
Centro Leste (NRS - Feira Santana) & 0 & 0 & 0 & 0 & 1 & 1 & 1 & 1 \\
Centro Norte (NRS - Jacobina) & 0 & 1 & 1 & 1 & 1 & 1 & 1 & 1 \\
\hline
\end{tabular}

NRS - Núcleo regionais de saúde /TAN - Triagem auditiva neonatal.

Fonte: Sistema de Informação Ambulatorial (SIA/SUS).

No que tange o quantitativo de equipamentos para a pesquisa do potencial evocado auditivo de tronco encefálico automático, por ano, na macrorregião do Estado da Bahia, observou que a grande maioria das macror- regiões de saúde da Bahia não tem este equipamentos, sendo a macrorregião Leste (NRS - Salvador) com a maior quantidade acumulado ao longo do período 2011-2018. (tabela 5)

Tabela 5 - Número de equipamentos do potencial evocado auditivo de tronco encefálico automático por ano na macrorregião do Estado da Bahia no período de 2011-2018.

\begin{tabular}{lcccccccc}
\hline \multicolumn{1}{c}{ Macrorregião de Saúde } & $\mathbf{2 0 1 1}$ & $\mathbf{2 0 1 2}$ & $\mathbf{2 0 1 3}$ & $\mathbf{2 0 1 4}$ & $\mathbf{2 0 1 5}$ & $\mathbf{2 0 1 6}$ & $\mathbf{2 0 1 7}$ & $\mathbf{2 0 1 8}$ \\
\hline Sul - (NBS - Ilhéus) & 0 & 1 & 1 & 2 & 2 & 2 & 2 & 2 \\
Sudoeste (NBS - Vitória da Conquista) & 0 & 0 & 0 & 0 & 0 & 0 & 0 & 0 \\
Oeste (NBS - Barreiras) & 0 & 0 & 0 & 0 & 0 & 0 & 1 & 1 \\
Norte (NRS - Juazeiro) & 0 & 0 & 0 & 0 & 0 & 0 & 0 & 0 \\
Nordeste (NRS - Alagoinhas) & 0 & 0 & 0 & 0 & 0 & 0 & 0 & 1 \\
Leste (NRS - Salvador) & 0 & 3 & 4 & 4 & 4 & 5 & 5 & 8 \\
Extremo Sul (NRS - Teixeira Freitas) & 0 & 0 & 0 & 0 & 0 & 0 & 1 & 2 \\
Centro Leste (NRS - Feira Santana) & 0 & 0 & 0 & 0 & 1 & 1 & 1 & 1 \\
Centro Norte (NRS - Jacobina) & 0 & 1 & 1 & 1 & 1 & 1 & 1 & 1 \\
\hline
\end{tabular}

NRS - Núcleo regionais de saúde /TAN - Triagem auditiva neonatal.

Fonte: Sistema de Informação Ambulatorial (SIA/SUS).

\section{DISCUSSÃO}

O interesse do presente estudo foi analisar a produção ambulatorial e a cobertura da TAN no território do Estado da Bahia após a vigência da lei no $12.303 / 2010$, sendo possível observar que a realização dos procedimentos da TAN, nas diferentes macrorregiões do Estado da Bahia, ainda encontra-se com níveis de cobertura insatisfatórias e desiguais apresentando taxas de crescimento anuais irregulares e inferiores ao preconizado pelo Comitê Multiprofissional em Saúde Auditiva (COMUSA, 2010).

A situação assimétrica de distribuição da cobertura da TAN no Estado da Bahia pode estar relacionada a sua extensão territorial, a qual ocupa a quinta posição de área do país (IBGE, 2019), bem como pela divergência de perfil socioeconômico e educacional dos usuários do SUS em cada macrorregião, fatores que contribuem para a baixa acessibilidade desse programa. Soma-se a isso a fragilidade de financiamento público para esses serviços, a falta de equipamentos e um quadro defasado de profissionais (CRUZ; FERRITI, 2014).

Um estudo de análise da cobertura da TAN no Nordeste demonstrou que os Estados de maiores extensões territoriais, inclusive a Bahia, tiveram uma evidente debilidade da concentração desses procedimentos de triagem (DIAS; PASCHOAL; CAVALCANTI, 2017).

Ainda em relação a cobertura dos procedimentos da TAN, verificou que as EOA foram responsáveis por mais de $90 \%$ da produção ambulatorial em detrimento do uso do PEATE. 
Essa dinâmica ocorre pelo fato das EOA serem os primeiros exames realizados na TAN de acordo com Diretrizes de Atenção da Triagem Auditiva Neonatal (2012) são as emissões otoacústicas transientes (EOAT). Essa escolha se dar por ser um exame de rápida execução, simples e de modo não invasivo que apresenta alta sensibilidade capaz de identificar a maioria das perdas auditivas cocleares em torno de 30-35 dB.

Nesse sentido, de acordo com as Diretrizes de atenção da TAN do Ministério da Saúde (2012), recomenda-se que o uso do PEATE - A seja realizado em casos de falhas no teste das EOA e naqueles indivíduos com indicação de risco para deficiência auditiva mesmo que tenham obtido resultados de "passa" nas EOA, panorama que pode explicar a considerável produção ambulatorial das EOA na TAN.

Entretanto, verifica-se que as macrorregiões Sul, Oeste, Norte, Nordeste, Extremo Sul, Centro Leste e Centro Norte da Bahia não registraram dados referentes a utilização do PEATE-A entre os anos de 2011 a 2018. Essa ausência de registro no DATASUS pode está relacionada a uma subnotificação por parte do serviço da TAN ou pela falta de equipamentos disponíveis nestes centros, sendo esta última a situação mais preocupante, uma vez que pode vir a comprometer a qualidade do programa e a cobertura universal da TAN na Bahia.

Visto que o PEATE - A é um procedimento de baixo custo com critérios de aprovação pautados por meio de análises estatísticas, a sua utilização antes da alta hospitalar em neonatos e lactantes contribui consideravelmente para a redução de taxas de retestes das EOAT, além de evitar os altos índices de evasão na continuação do processo de diagnóstico destes neonatos (CAVALCANTE et al., 2015) e também para reduzir a taxa de falso-positivo.

A importância da TAN não está apenas em realizar a triagem, mas também na detecção e intervenção precoce das perdas auditivas a fim de garantir um melhor desenvolvimento da fala e da linguagem, prevenindo dificuldades nas habilidades educacionais e socioemocionais (THE JOINT COMMITTEE ON INFANT HEARING, 2019).

Em contrapartida, a macrorregião Leste do Estado da Bahia concentra os maiores quantitativos de produção ambulatorial de procedimentos da TAN e as maiores taxas de cobertura do Estado, embora não alcancem a média nacional atual de coberta cujo indicador de universalidade da triagem encontra-se em torno de $37,2 \%$, assim como os seus índices são muito inferiores aos valores preconizados pelo Comitê Multiprofissional em Saúde Auditiva (COMUSA, 2010) juntamente com o Joint Committee on Infant Hearing (2000) que são superiores a 95\%.

Assim, essa situação sanitária contribui para as dificuldades de acesso ao serviço de assistência à saúde (PASCHOAL; CAVALCANTI; FERREIRA, 2016) nas suas macrorregiões, levando os usuários a migrar para outras regiões com maior suporte em assistência, como as capitais (a exemplo da macrorregião Leste - Salvador), o que ocasiona saturação nos centros de saúde especializados e aumento do custo por parte dos órgãos públicos locais responsáveis (BEVILACQUA et al., 2011).

Adicionalmente, ao analisar a distribuição dos equipamentos destinados a TAN em todas as macrorregiões da Bahia, nota-se também uma distribuição desigual das EOA e, na maioria das regiões, inexistência de registro de equipamentos para PEATE-A, fatores que comprometem a universalidade do programa da TAN, assim como vão contra a Lei que dispõe sobre a obrigatoriedade da realização gratuita desses exames em todos os hospitais e maternidades do território nacional.

Cita-se ainda, que muitas das macrorregiões que não apresentam registros de realização de alguns dos exames da TAN, em especial o PEATE-A, entre os anos 2011 a 2018 demonstraram ter sobre a sua dependência todos os equipamentos disponíveis para uso, o que sugere um provável mau uso desses equipamentos destinados a avaliação audiologica infantil nos centros públicos de saúde.

Embora o artigo tenha demonstrado a baixa cobertura da TAN nas diferentes macrorregiões de saúde no Estado da Bahia, os dados ainda não são suficientes para relatar as reais dificuldades enfrentadas para a consolidação da universalidade da TAN, porém servem de termômetro social que auxilia mensurar o desempenho dos serviços prestados aos usuários do SUS, tal como ajudam na definição de medidas estratégicas e planejamento de políticas públicas em saúde auditiva.

Outra limitação do estudo está relacionada ao fato de não ser possível distinguir os exames utilizados na etapa "teste" e "reteste" da TAN, portanto, definir quantas vezes um mesmo paciente realizou o exame, já que o DATASUS não disponibiliza um código para cada etapa, o que dificulta definir a frequência absoluta exata da produção ambulatorial da TAN.

Dessa forma, sugere-se que sejam realizados novos estudos nos aspectos de cobertura territorial e econômica no Estado da Bahia a fim de melhor evidenciar as desigualdades na distribuição de recursos para o serviço de saúde auditiva.

\section{CONCLUSÃO}

Os achados do presente estudo evidenciaram que os procedimentos da TAN não contemplam todas as macrorregiões de saúde do Estado da Bahia de forma igualitária, apresentando uma taxa de crescimento anual entre os anos 2011 a 2018 irregular, assim como um índice de cobertura dos serviços muito inferior a atual média nacional e, principalmente, ao preconizado pelos órgãos internacionais, o que compromete a universalização destes serviços no Estado da Bahia.

\section{REFERÊNCIAS}

BEVILACQUA, M. C. et al. Contribuições para análise da política de saúde auditiva no Brasil. Rev. Soc. Bras. Fonoaudiol., São Paulo, v.16, n.3, p.252-259, 2011. Disponível em: https://doi.org/10.1590/S151680342011000300004. Acesso em: 01 ago. 2020.

BRASIL. Lei n. 12.303, de 2 agosto de 2010. Dispõe sobre a obrigatoriedade de realização do exame denominado emissões 
otoacústicas evocadas. Câmara dos Deputados. Disponível em: http:// www.planalto.gov.br/ccivil_03/_Ato2007-2010/2010/Lei/L12303.htm. Acesso em: 10 jul. 2020.

BRASIL. Ministério da Saúde. Diretrizes de atenção da triagem auditiva neonatal. Brasília: MS, 2012. Disponível em: https://bvsms.saude.gov.br/bvs/publicacoes/diretrizes_atencao_ triagem_auditiva_neonatal.pdf. Acesso em: 15 jul. 2020.

CAVALCANTE, J. M. S et al. A importância do protocolo combinado em um programa de triagem auditiva neonatal universal. Disponível em: https://www.audiologiabrasil.org.br/eiabauru2015/anais2015/ resumos/R0123-1.PDF. Acesso em: 20 out. 2020.

CONSELHO FEDERAL DE FONOAUDIOLOGIA (CFF). Triagem Auditiva Neonatal Universal (TANU): implantação com ética, técnica e responsabilidade. 2019. Disponível em: https://www.fonoaudiologia. org.br/wp-content/uploads/2019/09/folder-tanu-1.pdf. Acesso em: 14 set. 2020.

CONSELHO REGIONAL DE FONOAUDIOLOGIA 4 REGIÃO (CREFONO 4). Crefono participa de debate no ministério público da Bahia sobre triagem neonatal. Disponível em: http://www.crefono4.org.br/noticias/ noticia/1523/crefono-participa-de-debate-no-ministerio-publico-dabahia-sobre-triagem-neonatal. Acesso em: 06 jul.20.

CRUZ, L. R. L.; FERRITE, S. Cobertura estimada da triagem auditiva neonatal para usuários do Sistema Único de Saúde, Brasil, 20082011. Rev. bras. saúde mater. infant., Recife, v.4, n.4, p.401-11, 2014. Disponível em: https://doi. org/10.1590/S1519-38292014000400010. Acesso em: 26 ago. 2020

DIAS, W.C.F.G.S.; PASCHOAL, M.R.; CAVALCANTI, H.G. Análise da cobertura da triagem auditiva neonatal no Nordeste brasileiro. Audiol. Commun. Res., São Paulo, v. 22, p. 2-5, 2017. Disponível em: https://doi. org/10.1590/2317-6431-2017-1858. Acesso em: 03 set. 2020.

INSTITUTO BRASILEIRO DE GEOGRAFIA E ESTATÍSTICA (IBGE). Disponível em: https://cidades.ibge.gov.br/brasil/ba/salvador/panorama. Acesso em: 19 out. 2020.

JOINT COMMITTEE ON INFANT HEARING. Year 2000 position statement: principles and guidelines for early hearing detection and intervention programs. J. Pediatr., Rio de Janeiro, v.106, p.798- 817, 2000.

LEWIS, D. R. et al. Comitê Multiprofissional em Saúde Auditiva (COMUSA). Braz. j. otorhinolaryngol., São Paulo, v.76, n.1, p.121-128, 2010. Disponível em: https://doi.org/10.1590/S1808- 86942010000100020. Acesso em: 20 ago. 2020.

PASCHOAL, M. R.; CAVALCANTI, H. G.; FERREIRA, M.A.F. Análise espacial e temporal da cobertura da triagem auditiva neonatal no Brasil (2008-2015). 2016. Dissertação (Mestrado) - Universidade Federal do Rio Grande Norte, Natal, 2016. Disponível em: https://doi. org/10.1590/1413-812320172211.21452016. Acesso em: 15 jul. 2020.

SIH, T.; GODINHO, R. A criança com perda auditiva e surdez: o papel da família e do pediatra. SBP, 2018. Disponível em: https://www.sbp. com.br/fileadmin/user_upload/ARTIGO_A_CRIANCA_COM_PERDA_ AUDITIVA_E_SURDEZ_9_NOVEMBRO_2018.pdf. Acesso em: 24 jul. 2020.

THE JOINT COMMITTEE ON INFANT HEARING. Year 2019 Position Statement: principles and guidelines for early hearing detectionand intervention programs. J. Early Hear. Detect. Interv., [s.I], v.4, n. 2, p.1-44, 2019. Disponível em: https://digitalcommons.usu.edu/cgi/viewcontent. cgi ?article=1104\&context=jehdi. Acesso em: 20 ago. 2020.

Submetido em: $07 / 12 / 2020$

Acesso em: 14/12/2020 